\section{Rule of three}

At the August meeting of the American Chemical Society in Washington, DC, Zhaochun Ma and John-Stephen Taylor of Washington University (St. Louis, MO) reported a strategy that uses disease-specific gene sequences to activate cytotoxic drugs. Their system employs two oligonucleotides, one that binds to a disease-specific trigger sequence and is coupled to an inactive prodrug, and the other that binds to an adjacent sequence and is coupled to a pro-drug activator. They used imidazole to release the cytotoxic drug $p$-nitrophenyl from its ester, a reaction that is a key step in activation of a recently reported class of daunorubicin pro-drugs. The presence of all three components triggered formation of a complex that brought the prodrug in contact with the catalytic activator. The prodrug was then activated enzymatically and in multiple cycles, but in the presence of a single base pair mismatch, drug release was 7.5-fold less efficient. Taylor cautions that "We have yet to determine whether the prodrug releasing system is stable in plasma and inside cells and efficient enough....This might be the most challenging part of the work."

ND

\section{Chromosome targeting}

Scientists have literally thrown light on the problem of gene targeting. Using a two-photon scanning laser microscope, scientists at the University of California Irvine and the University of California San Diego have performed targeted gene inactivation in cultured cells (PNAS 97, 9504-9507, 2000). Using a conveniently flat line of cultured PTK2 cells, the researchers were able to visualize the chromosomal loci containing the ribosomal DNA. A DNA-associating dye was added to the cells to make the chromosomes photosensitive, and the ribosomal DNA locus was inactivated by precisely aiming the focal point of the twophoton microscope. All of the cells survived the treatment, which reliably knocked out the targeted locus. The authors argue that existing techniques, such as fluorescence in situ hybridization, should allow the same technique to be used on virtually any gene for which specific probes are available. Michael Berns, first author on the paper, says that the multiphotontargeted gene inactivation approach "could be used on most standard two-photon microscopes," allowing researchers to apply it almost immediately.

$A D$

Research News Briefs written by Aaron J. Bouchie, Natalie DeWitt, and Alan Dove.

\title{
Roll out the barrel scaffold
}

Work on $(\beta \alpha)_{8}$-barrel proteins by a team at the European Molecular Biology Laboratory (EMBL, Hamburg, Germany) could provide insights into strategies for engineering new enzyme activities. In a study in Science (289, 1546-1550, 2000), the EMBL team compared atomic structures of a group of related enzymes of the histidine biosynthesis pathway, HisA and HisF, and of the tryptophan biosynthesis pathway, $\operatorname{TrpC}$, TrpF, and TrpA. Their analysis indicates that these enzymes evolved by gene duplication and diversification from an ancestral $(\beta \alpha)_{8}$-barrel, itself the product of gene

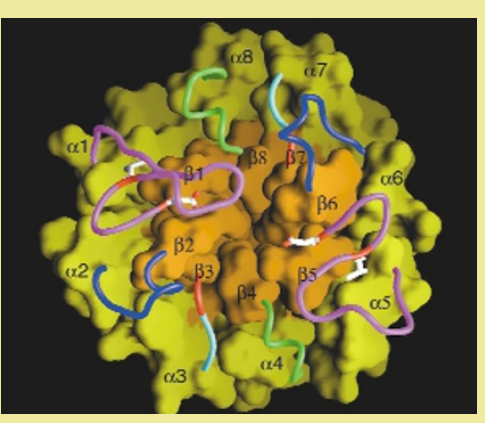
duplication and fusion of a $(\beta \alpha)_{4}$-half barrel. Using random mutagenesis and selection, they also show that variants of HisA could be generated to catalyze the same reaction as TrpF, one of which retained its His A activity as well (PNAS 97, 9925-9930, 2000). They suggest that a similar enzyme could have been an evolutionary precursor of both HisA and TrpF, although the possibility of TrpF evolving directly from HisA cannot be ruled out. The findings "should inspire other research into apparent single-domain proteins to discover they have also arisen by gene duplication," speculates Stanford (CA) molecular biologist Charles Yanofsky. If successful, the work could be exploited to create new enzymes of industrial interest.

$A J B$

\section{Bt corn kills monarch?}

A new study published online August 19 in Oecologia (http://link.springer.de/link/service/journals/00442/) provides evidence that pollen from $B t$ transgenic corn kills larvae of the monarch butterfly, Danaus plexippus. This contradicts earlier fieldwork that suggested nontarget lepidopteran larvae were unaffected by $B t$ corn pollen (Nat. Biotechnol. 18, 701, 2000). The twoyear Iowa State (Ames, IA) study compared the effects of two Novartis Seeds (Golden Valley, MN) transgenic corn strains against genetically similar nontransgenic hybrids. When monarchs fed on milkweed (their natural food source) for $48 \mathrm{~h}$ within 10 meters of the transgenic cornfield plots, $20 \pm 3 \%$ mortality was observed, as opposed to no mortality when fed near nontransgenic corn. Pollen densities were measured (1-217 pollen grains $/ \mathrm{cm}^{2}$ ) and mortality was observed on leaves with as few as 10 transgenic pollen grains $/ \mathrm{cm}^{2}$. When the experiment was simulated in the laboratory, 135 pollen grains $/ \mathrm{cm}^{2}$ resulted in mortality levels similar to field treatments. However, transgenic corn pollen had no effect on surviving butterflies' development time, wing length, or lipid content. The data suggest that $B t$ corn can affect the survival of nontarget lepidopteran insects up to $10 \mathrm{~m}$ from cornfields, although certain experts have reservations about the data (see p. 1031).

\section{A snare for the weak}

Researchers have reported a powerful new screen for identifying weak binders to specific proteins. The new technique, described by researchers at Sunesis Pharmaceuticals (Redwood City, CA) and the University of California (San Francisco, CA), should be especially useful for identifying starting fragments for rational drug design. Standard screening approaches for smallmolecule inhibitors are limited to identifying compounds with relatively high affinities for the target protein, and do not allow selection for specific target sites. In the new procedure (PNAS 97, 9367-9372, 2000), the disulfide-containing compounds being screened can form a covalent linkage with the protein. A cysteine residue in the protein will "tether" any compound that has even a moderate affinity for the nearby active site, allowing the compound to be identified by mass spectrometry. The team demonstrated the concept by identifying an inhibitor of thymidylate synthase. After identifying an inhibitor from the initial library, the scientists improved its affinity by several orders of magnitude through rational design. James Wells, senior author on the paper, says extending the approach to a wide range of targets should be straightforward: "Enzyme targets are very exciting, and we're also going after protein-protein targets, which have been virtually intractable to drug screening." ND 